\title{
Short communication: Sodium salicylate negatively affects rumen fermentation in vitro and in situ
}

\author{
A. J. Carpenter, ${ }^{1,2}$ C. F. Vargas Rodriguez, J. A. B. Jantz, and B. J. Bradford \\ Department of Animal Sciences and Industry, Kansas State University, Manhattan 66506
}

\begin{abstract}
Administration of sodium salicylate (SS) to cows in early lactation has a positive effect on whole-lactation milk production but a negative effect on metabolism in some cases. The objective of this trial was to determine whether SS directly affects rumen fermentation. Experiment 1 was designed to investigate the effects of direct inclusion of SS in a 24-h batch culture, and experiment 2 was designed to test the fermentative ability of rumen fluid from heifers who had received SS. In experiment 1 , we combined strained and pooled rumen fluid from 3 heifers in a 2:1 ratio with McDougall's buffer, and added $150 \mathrm{~mL}$ of the inoculum to each flask $(\mathrm{n}=5$ / treatment) with $2.5 \mathrm{~g}$ of fermentation substrate similar to a lactating cow ration, ground to $1 \mathrm{~mm}$. We then added premixed treatments (1-mL volume) to achieve the desired final amount of SS $(\mathrm{CON} 1=0 \mathrm{mg}$, LOW $=$ $125 \mathrm{mg}, \mathrm{MED}=250 \mathrm{mg}, \mathrm{HI}=375 \mathrm{mg}$ ). In experiment 2,6 heifers $(\mathrm{n}=3 /$ treatment $)$ were drenched daily for $3 \mathrm{~d}$, either with $62.5 \mathrm{~g}$ of SS dissolved in water (SAL) or an equal volume of water (CON2). Rumen fluid was collected from each heifer and was not pooled. After the fluid was mixed 2:1 with McDougall's buffer, $150 \mathrm{~mL}$ of inoculum was added to the fermentation flasks (n $=4 /$ heifer) with $2.5 \mathrm{~g}$ of fermentation substrate. This experiment was performed the day before SS treatment began and repeated 1, 13, and $35 \mathrm{~d}$ after the end of the treatment period. We also performed an in situ experiment at each of these time points. In the first experiment, inclusion of SS resulted in a decrease in dry matter disappearance (DMD) over $24 \mathrm{~h}$, as well as an increase in final $\mathrm{pH}$. We detected no difference between treatments for gas production asymptotic volume, rate, or lag. In the second experiment, we detected a significant treatment $\times$ day interaction for DMD: we observed no difference between groups during a 24-h
\end{abstract}

Received August 5, 2016.

Accepted November 9, 2016.

${ }^{1}$ Current affiliation: Department of Animal Biosciences, University of Guelph, Ridgetown, ON, N0P 2C0, Canada.

${ }^{2}$ Corresponding author: acarpenter@uoguelph.ca batch culture on the day following treatment, but SAL resulted in lower DMD on d 13 and d 35. We detected no treatment effect on the final $\mathrm{pH}$ of the batch culture or on any gas-production parameters. We observed a tendency for SAL to decrease the DMD rate in situ on the day after treatment. These results indicate that SS administration has a negative effect on rumen microorganisms.

Key words: rumen fermentation, sodium salicylate, in vitro

\section{Short Communication}

During transition into lactation after calving, dairy cattle experience elevated systemic inflammation. Administration of the anti-inflammatory medication sodium salicylate (SS) after calving has been shown to increase whole-lactation milk production in multiparous dairy cows, but treatment with SS has been associated with hypoglycemia in some circumstances. Farney et al. (2013a) reported that cows had decreased blood glucose concentrations after receiving SS in their drinking water for $7 \mathrm{~d}$ after calving, but 305-d milk yield was greater in older cows (parity 3 and greater) that received SS compared with controls (Farney et al., 2013b). In a follow-up study, Carpenter et al. (2016b) demonstrated that giving 3 daily doses of SS after calving in multiparous cows did not result in the same hypoglycemia, but 305-d milk yield was still increased.

Salicylic acid functions as a hormone in plants to combat pathogens. It has anti-inflammatory properties in mammals via its interactions with the nuclear factor (NF)-KB pathway, which is involved with gene expression during inflammation and infection (Kopp and Ghosh, 1994). Besides SS, other forms of salicylate have also been shown to be antimicrobial, with the ability to depress rumen microbial function. Ruiz-Moreno et al. (2015) administered bismuth subsalicylate (BSS) to rumen microbes in a batch culture in an attempt to reduce hydrogen sulfide production resulting from the fermentation of distillers grains. When BSS was included at 2 and $4 \%$ of $\mathrm{DM}$, final $\mathrm{pH}$ was increased, and total VFA concentration was decreased, at $4 \%$ of 
Table 1. Ingredient and chemical composition of fermentation substrate for in vitro rumen fermentation experiments

\begin{tabular}{lc}
\hline Item & \% of DM \\
\hline Corn silage & 22.0 \\
Alfalfa hay & 21.0 \\
Corn grain & 25.0 \\
Cottonseed & 4.0 \\
Dried distillers grains & 14.0 \\
Soybean meal & 14.0 \\
CP & 21.5 \\
NDF & 24.8 \\
ADF & 18.9 \\
Ether extract & 3.9 \\
\hline
\end{tabular}

DM. When BSS was added during continuous culture fermentation at $1 \%$ of DM, total VFA concentrations were also decreased, and $\mathrm{pH}$ and digestion of $\mathrm{OM}$, NDF, and ADF were increased. Similarly, Fessenden (2013) reported that when BSS was administered at 0 or $0.5 \%$ of diet DM (at 2 different levels of sulfur), total VFA concentrations were decreased and $\mathrm{pH}$ was increased, and OM digestion was also decreased.

Experiments with human colonic bacteria have also shown an antimicrobial effect of BSS. In the stomach, BSS is hydrolyzed to form salicylic acid (Bierer, 1990). Salicylate is believed to be partially responsible for the antibacterial effects of BSS. Cornick et al. (1990) reported that although SS was as active as BSS against aerobic bacteria, it was not as active against anaerobic bacteria, such as those found in the rumen, although inhibitory activity was still observed. León-Barúa et al. (1990) demonstrated that BSS reduced gas production by colonic bacteria in vitro to a greater extent than other bismuth-containing compounds. Additionally, Manhart (1990) showed a dose-dependent inhibition of various strains of bacteria to SS.

The objective of these experiments was to determine the effect of SS on in vitro fermentation by rumen microorganisms. In the first experiment, SS was directly included in a dose-dependent manner in batch cultures of rumen bacteria. In the second experiment, SS was administered to heifers, and the rumen fluid collected from these animals was tested for its fermentative ability in batch culture.

In experiment 1, SS was added directly to batch cultures of rumen fluid at different amounts (CON1 $=0 \mathrm{mg}, \mathbf{L O W}=125 \mathrm{mg}, \mathbf{M E D}=250 \mathrm{mg}, \mathbf{H I}=$ $375 \mathrm{mg}$ ). Rumen fluid was collected from 3 heifers, strained through 8 layers of cheesecloth immediately following collection and 4 layers of cheesecloth immediately before flocculation, and allowed to incubate in the laboratory for approximately $1 \mathrm{~h}$ to allow it to stratify. The bottom layer was discarded, and the middle layer was collected for pooling. Pooled fluid was combined in a 2:1 ratio with McDougall's buffer, and $150 \mathrm{~mL}$ of the inoculum was added to each $250-\mathrm{mL}$ flask (n $=5 /$ treatment). Five blank flasks contained inoculum alone, and each treatment and control flask contained $2.5 \mathrm{~g}$ of fermentation substrate (Table 1). Before inoculum was added to the flasks, $1 \mathrm{~mL}$ of premixed treatment was added to achieve the desired final amount of SS. Cumulative gas pressure was measured using the Ankom $^{R F}$ Gas Production System (Ankom Technology, Macedon, NY). Vessel pressure was recorded at $5 \mathrm{~min}$ intervals. Vessels were incubated at $39.5^{\circ} \mathrm{C}$ for $24 \mathrm{~h}$. Following incubation, fermentation was inhibited by placing fermentation vessels on ice. At this time, final $\mathrm{pH}$ was measured and fermentation vessels were emptied into individual containers and dried at $60^{\circ} \mathrm{C}$ for 48 h. Dry matter disappearance (DMD) was quantified as the proportion of dry matter remaining, less the approximate contribution of DM from the rumen fluid (estimated by final dry weight of the content of the blank flasks) as a proportion of the initial DM of the substrate.

In experiment 2, 6 heifers [age $=10$ mo, standard deviation $(\mathrm{SD})=0.3 ; \mathrm{BW}=614.2 \mathrm{~kg}, \mathrm{SD}=44.87$; $\mathrm{n}=3$ /treatment) were drenched daily for $3 \mathrm{~d}$ with either $62.5 \mathrm{~g}$ of SS in water (SAL) or an equal volume of water (CON2) approximately $12 \mathrm{~h}$ after feeding. Each heifer received the same high-forage ration $(\mathrm{CP}=$ $11.9 \%$ of $\mathrm{DM} ; \mathrm{NDF}=48.5 \%$ of $\mathrm{DM} ; \mathrm{ADF}=32.3 \%$ of DM; ether extract $=2.5 \%$ of DM). Four batch cultures were performed as described above with the exception that rumen fluid was not pooled and SS was not added to the inoculum, such that heifer was the experimental unit. Rumen fluid was collected approximately $14 \mathrm{~h}$ after the final dose of SS. Inoculum from each heifer was replicated in 4 flasks with 2.5 g substrate added, and 2 flasks without substrate functioned as blanks for each heifer. Batch cultures were performed on the day before the start of treatment, as well as at 1, 13, and $35 \mathrm{~d}$ following treatment. During each batch culture, heifers were handled in blocks containing $1 \mathrm{CON} 2$ and $1 \mathrm{SAL}$ animal, in an attempt to prevent bias between treatments due to handling.

An in situ experiment was performed in parallel with experiment 2 to estimate the rate of substrate degradation in the rumen. Immediately following each rumen fluid collection, we inserted 2 Dacron bags (5 $\times 10 \mathrm{~cm} ; 50 \mu \mathrm{m}$ porosity) containing approximately $1 \mathrm{~g}$ of substrate DM (Table 1) into the rumen of each heifer for each time point. Time points before removal of the bags from the rumen were 2, 8, 16, 24, and $48 \mathrm{~h}$. Additionally, 12 bags were rinsed under running water and washed with the other bags upon removal from the rumen to estimate solubility. After rinsing or diges- 
tion, bags were dried in a forced-air oven at $105^{\circ} \mathrm{C}$ and weighed to measure the remaining dry matter.

In both experiments, we estimated gas variablesasymptotic volume, lag, and rate - using the NLIN procedure of SAS 9.3 (SAS Institute Inc., Cary, NC), which obtains estimates using nonlinear least squares. We used the following model to obtain estimates:

$$
x=\frac{v}{1+e^{[2-4 k(t-l)]},}
$$

where $v=$ total volume of gas produced $(\mathrm{mL}), k=$ rate of gas production $(\mathrm{mL} / \mathrm{min}), t=$ time $(\mathrm{min})$, and $l=$ lag in gas production (min). After obtaining estimates for each flask, gas-production variables were analyzed for each experiment as described for other variables.

Data from experiment 1 were analyzed using the GLM procedure of SAS, with treatment as the independent variable and gas production metrics, DMD, final $\mathrm{pH}$, and the change in $\mathrm{pH}$ as dependent variables. Data from experiment 2 were analyzed using the MIXED procedure of SAS. Each dependent variable was analyzed with its own value on the first day of the experiment (baseline) as a covariate. Besides these covariates, the model included the fixed effects of treatment, day, and treatment $\times$ day interaction. The random statement contained heifer and replicate within heifer, and repeated measures were used across days within heifer. An autoregressive(1) covariance structure was selected based on Bayesian information criterion value.

In experiment 1, DMD was depressed by the inclusion of SS $(P<0.05)$ : HI had lesser DMD than LOW $(P<0.05)$, and MED was intermediate (Table 2$)$. Final $\mathrm{pH}$ was similar for LOW and CON1, but MED and HI had a higher final $\mathrm{pH}$ than CON1 $(P<0.05$; Table $2)$. We observed no differences in gas production for asymptotic volume, rate, or lag $(P \geq 0.28$; Table 2$)$.

The results for experiment 2 are reported in Table 3. We observed no influence of SAL on the final $\mathrm{pH}$ of batch cultures at any time point $(P=0.71)$. The final $\mathrm{pH}$ of the batch culture performed before treatment administration was a significant predictor of final $\mathrm{pH}$ at all time points after treatment $(P=0.03)$. Immediately after treatment, SAL had no effect on DMD $(P=0.67)$; however, treatment reduced $(P<0.01)$ DMD in batch culture $13 \mathrm{~d}$ after treatment, and DMD was still reduced $(P=0.01) 35 \mathrm{~d}$ after SAL treatment (treatment $\times$ day: $P=0.01)$. For the in situ portion of experiment 2 , we detected no differences due to treatment for rate of DMD $(P=0.21)$. We observed a significant effect of treatment and day (both $P \leq 0.01$ ) on DMD at $48 \mathrm{~h}$ (treatment $\times$ day: $P=0.31$ ). Based on these results, it would appear that SS treatment causes long-term inhibition of rumen fermentation or digestion.

As discussed above, other salicylate compounds-specifically BSS - have negative effects on rumen fermentation. Our findings are similar to those of Ruiz-Moreno et al. (2015), who showed that BSS inclusion at 2 and $4 \%$ of DM in batch cultures increased final $\mathrm{pH}$ at $24 \mathrm{~h}$. In continuous culture, inclusion of BSS at $1 \%$ of DM increased average $\mathrm{pH}$, although digestion of $\mathrm{OM}$ was also increased (Ruiz-Moreno et al., 2015). Similar to the current study, however, Fessenden (2013) reported that inclusion of BSS at $0.5 \%$ of DM in continuous culture decreased true and apparent DM and OM digestion, with a corresponding increase in average $\mathrm{pH}$. For comparison, the present experiment included SS at approximately 5, 9, and $13 \%$ of substrate DM for LOW, MED, and HI, respectively. It should be noted, however, that BSS is a larger molecule than SS, at a molecular weight of $362.09 \mathrm{~g} / \mathrm{mol}$, compared with $160.10 \mathrm{~g} / \mathrm{mol}$ for SS. Salicylic acid itself has a molecular weight of 138.12 $\mathrm{g} / \mathrm{mol}$. Therefore, the salicylate component of BSS is roughly $38 \%$ of its molecular mass, but it composes approximately $85 \%$ of the SS molecule. Based on these calculations, BSS at 2 or $4 \%$ of DM is approximately equivalent to salicylate inclusion at 0.8 or $1.5 \%$ of $\mathrm{DM}$, respectively, but SS levels at 5, 9, and $13 \%$ of DM is approximately equivalent to salicylate inclusion at 4,8 , and $11 \%$ of DM, respectively. If in vitro substrate DM is considered to be roughly the equivalent of DMI, this level of inclusion would be much higher than recommended in vivo. However, this assumes that the ratio

Table 2. Effects of adding sodium salicylate ${ }^{1}$ at different amounts on fermentation by rumen microbes in vitro for $24 \mathrm{~h}$ (experiment 1 )

\begin{tabular}{|c|c|c|c|c|c|}
\hline Item & CON1 & LOW & MED & HI & Pooled SEM \\
\hline 24-h disappearance (\% of DM) & $48.8^{\mathrm{a}}$ & $37.08^{\mathrm{b}}$ & $29.59^{\mathrm{bc}}$ & $22.78^{\mathrm{c}}$ & 1.96 \\
\hline \multicolumn{6}{|l|}{ Gas-production measurements } \\
\hline Rate $(\mathrm{mL} / \mathrm{min})$ & 0.0010 & 0.0010 & 0.0008 & 0.0010 & 0.0002 \\
\hline $\operatorname{Lag}(\min )$ & 137.0 & 122.2 & 79.1 & 127.8 & 55.09 \\
\hline
\end{tabular}

\footnotetext{
${ }^{\mathrm{a}-\mathrm{c}}$ Means within a row with different superscripts differ $(P<0.05)$.

${ }^{1}$ Sodium salicylate was added to rumen fluid inoculum at the beginning of a 24 -h batch culture of mixed rumen microbes in buffer; CON1 $=0$ $\mathrm{mg}$ of sodium salicylate, $\mathrm{LOW}=125 \mathrm{mg}, \mathrm{MED}=250 \mathrm{mg}, \mathrm{HI}=375 \mathrm{mg} ; \mathrm{n}=5$ flasks $/$ treatment.
} 
Table 3. Effects of drenching heifers with sodium salicylate ${ }^{1}$ on the fermentation capacity of rumen microbes in vitro for $24 \mathrm{~h}$ and in situ (experiment 2)

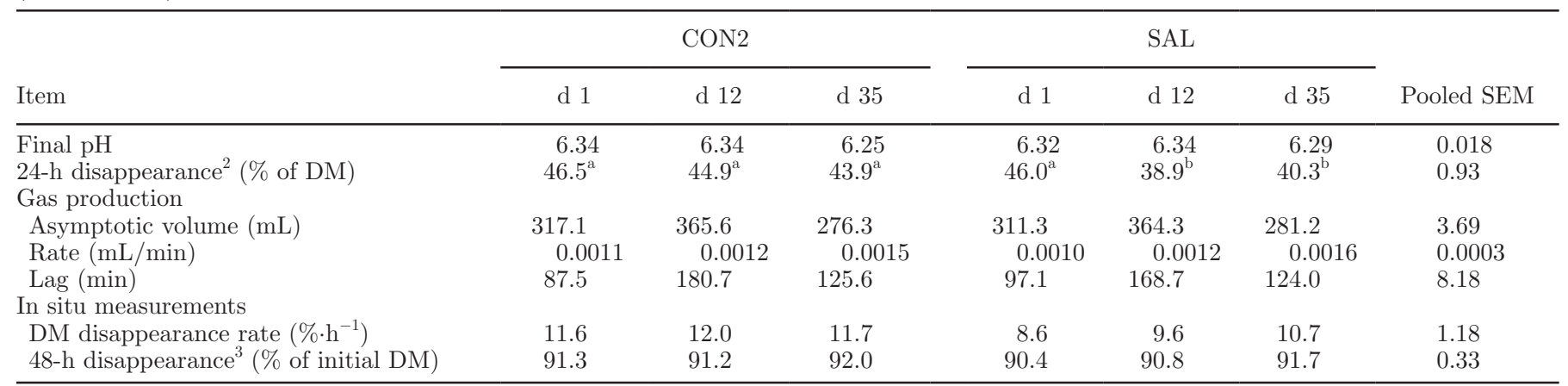

${ }^{\mathrm{a}, \mathrm{b}}$ Means within a row with different superscripts differ $(P<0.05)$.

${ }^{1}$ Heifers were drenched with $62.5 \mathrm{~g}$ of sodium salicylate (SAL) or water (CON2) for $3 \mathrm{~d}$, and in vitro and in situ experiments were conducted at 1,12 , and $35 \mathrm{~d}$ after treatment administration to test the ability of rumen microorganisms to ferment substrate.

${ }^{2}$ Means differed due to treatment $(P<0.01)$, day $(P<0.01)$, and the interaction between treatment and day $(P=0.01)$.

${ }^{3}$ Means differed due to treatment $(P=0.01)$ and day $(P<0.01$; treatment $\times$ day: $P=0.31)$.

of substrate to volume in vitro is roughly equivalent to the ratio of digesta to volume in the rumen, which is not the case. Alternatively, the ratio of SS to volume in the flasks for MED was roughly equivalent to 125 $\mathrm{g}$ of SS in a rumen volume of $65 \mathrm{~L}$, meaning that the SS concentration in the solution was at approximately physiological levels for this treatment. Regardless, the results of experiment 2 indicate an effect of SS administration on the ruminal environment at physiologically relevant levels.

Unlike the results presented here, Ruiz-Moreno et al. (2015) reported a significant decrease in gas production during 24-h batch culture with increasing levels of BSS inclusion. This was likely due to differences in measurement of gas production. Ruiz-Moreno et al. (2015) measured gas production by displacement of water when batch cultures were performed in serum bottles; the present study used the Ankom ${ }^{R F}$ system. We hypothesize that the simplistic measurement used by RuizMoreno et al. (2015) reduced measurement variation compared with the present experiment, increasing the statistical power of gas-production measurement.

These results appear to be counterintuitive to previous accounts that showed an increase in milk production in response to SS treatment in early lactation, but they may help to explain the metabolic outcomes observed in these studies. Despite the positive effects on milk production observed in older cows, cows in their second and higher parity experienced hypoglycemia in early lactation under certain experimental conditions (Farney et al., 2013a). This coincided with a higher value in the revised quantitative insulin sensitivity check (RQUICKI; an estimate of relative insulin sensitivity (Holtenius and Holtenius, 2007) on d 7 of treatment without any differences in expression of rate-determining hepatic gluconeogenic enzymes. It is feasible that this observation was a result of decreased production of glucogenic precursors in the rumen, possibly in addition to the effects of SS on insulin sensitivity. In fact, decreased substrate availability in the liver may have served to enhance insulin sensitivity in these animals. Bjerre-Harpøth et al. (2012) reported that cows in early lactation who underwent feed restriction experienced a significant change in RQUICKI values and had a higher ratio of glucagon to insulin, potentially indicating enhanced insulin sensitivity. Lower amounts of propionate from ruminal fermentation due to depression in microbial activity or changes in bacterial community composition could have the same effect.

Precedents can be found in the literature for sustained effects after modification of the microbial population, but to our knowledge, this experiment was unique in the length of time we observed a difference after treatment administration. Weimer et al. (2010b) noted that bacterial community composition did not completely return to its original state up to 4 wk after monensin was removed in combination with a milk-fat-inducing diet. Although exceptions such as this exist, it is difficult to force shifts in the rumen microbial population for an extended period of time (Weimer, 1998). Even after almost complete exchange of rumen contents, microbial populations in the rumen demonstrate a specificity for the host that is difficult to overcome by non-host forces (Weimer et al., 2010a). This is why experiments using a Latin square design to study rumen modifiers can successfully implement a wash-out period to minimize carry-over effects. Considering this information, the results of the current experiment are surprising. 
Other research has shown long-term alterations in feeding behavior in response to SS administration in lactating cows (Carpenter et al., 2016a). It is likely that these observations and the findings of the present experiments are related, but further research is needed to determine the cause-and-effect relationship between ruminal fermentation and feeding behavior after SS treatment. Some programming effect of the rumen microbial population may have resulted in a long-term shift that changed the fermentative ability of the rumen, but forces outside of the rumen (such as a neurological effect) could also have altered feeding behavior, in turn changing the rumen environment and resulting in a population shift. Unfortunately, we did not collect data on DMI and feeding behavior for the heifers used in experiment 2.

Although these experiments were relatively simple, they strongly suggest an antimicrobial effect of SS on rumen microorganisms. We observed not only an immediate and dose-dependent effect of this compound in vitro, but also a sustained negative effect on the ability of rumen microorganisms to degrade DM. Several questions remain. We did not perform an analysis on VFA production or profile in vitro or in vivo, nor did we characterize microbial populations following SAL in experiment 2. Future research should focus on these questions, as well as the effects on the rumen in vivo. It is unclear why a sustained positive response in milk production has been observed following SS administration in early lactation when the evidence here indicates that rumen function is hampered. Future research to explore this relationship, as well as the relationship between SS and feeding behavior, is clearly warranted.

\section{ACKNOWLEDGMENTS}

Contribution no. 17-232-J from the Kansas Agricultural Experiment Station. This project was supported by Agriculture and Food Research Initiative Competitive Grant no. 2013-01976 from the USDA National Institute of Food and Agriculture (Washington, DC). The authors thank Mike Scheffel (Manhattan, KS) and staff of the Kansas State Dairy Teaching and Research Center for their contributions to this work.

\section{REFERENCES}

Bierer, D. W. 1990. Bismuth subsalicylate: History, chemistry, and safety. Rev. Infect. Dis. 12(Suppl. 1):S3-S8.

Bjerre-Harpøth, V., N. C. Friggens, V. M. Thorup, T. Larsen, D. M. Damgaard, K. L. Ingvartsen, and K. M. Moyes. 2012. Metabolic and production profiles of dairy cows in response to decreased nutrient density to increase physiological imbalance at different stages of lactation. J. Dairy Sci. 95:2362-2380.

Carpenter, A. J., C. M. Ylioja, and B. J. Bradford. 2016a. Early postpartum administration of sodium salicylate to multiparous dairy cattle is associated with alterations in feeding behavior up to 120 d in milk. J. Dairy Sci. 99(E-Suppl. 1):521. (Abstr.)

Carpenter, A. J., C. M. Ylioja, C. F. Vargas, L. K. Mamedova, L. G. Mendonça, J. F. Coetzee, L. C. Hollis, R. Gehring, and B. J. Bradford. 2016b. Hot topic: Early postpartum treatment of commercial dairy cows with nonsteroidal antiinflammatory drugs increases whole-lactation milk yield. J. Dairy Sci. 99:672-679.

Cornick, N. A., M. Silva, and S. L. Gorbach. 1990. In vitro antibacterial activity of bismuth subsalicylate. Rev. Infect. Dis. 12(Suppl. 1):S9-S10.

Farney, J. K., L. K. Mamedova, J. F. Coetzee, B. KuKanich, L. M. Sordillo, S. K. Stoakes, J. E. Minton, L. C. Hollis, and B. J. Bradford. 2013a. Anti-inflammatory salicylate treatment alters the metabolic adaptations to lactation in dairy cattle. Am. J. Physiol. Regul. Integr. Comp. Physiol. 305:R110-R117.

Farney, J. K., L. K. Mamedova, J. F. Coetzee, J. E. Minton, L. C. Hollis, and B. J. Bradford. 2013b. Sodium salicylate treatment in early lactation increases whole-lactation milk and milk fat yield in mature dairy cows. J. Dairy Sci. 96:7709-7718.

Fessenden, S. W. 2013. Effects of bismuth subsalicylate and beta extract of hops (Humulus lupulus) on in vitro fermentation with ruminal microbes. MS Thesis. University of Minnesota, Minneapolis.

Holtenius, P., and K. Holtenius. 2007. A model to estimate insulin sensitivity in dairy cows. Acta Vet. Scand. 49:29-31.

Kopp, E., and S. Ghosh. 1994. Inhibition of NF-KB by sodium salicylate and aspirin. Science 265:956-959.

León-Barúa, R., R. Tello, M. del Carmen Morante, M. Alvarez, R. H. Gilman, and W. M. Spira. 1990. In vitro and in vivo effects of three bismuth compounds on fermentation by colonic bacteria. Rev. Infect. Dis. 12(Suppl. 1):S24-S29.

Manhart, M. D. 1990. In vitro antimicrobial activity of bismuth subsalicylate and other bismuth salts. Rev. Infect. Dis. 12(Suppl. 1):S11-S15.

Ruiz-Moreno, M., E. Binversie, S. W. Fessenden, and M. D. Stern. 2015. Mitigation of in vitro hydrogen sulfide production using bismuth subsalicylate with and without monensin in beef feedlot diets. J. Anim. Sci. 93:5346-5354.

Weimer, P. J. 1998. Manipulating ruminal fermentation: A microbial ecological perspective. J. Anim. Sci. 76:3114-3122.

Weimer, P. J., D. M. Stevenson, H. C. Mantovani, and S. L. C. Man. 2010a. Host specificity of the ruminal bacterial community in the dairy cow following near-total exchange of ruminal contents. J. Dairy Sci. 93:5902-5912.

Weimer, P. J., D. M. Stevenson, and D. R. Mertens. 2010b. Shifts in bacterial community composition in the rumen of lactating dairy cows under milk fat-depressing conditions. J. Dairy Sci. 93:265278. 